\title{
Establishing patient partners: The Health Care
} Health Design Home model

\section{DESIGN}

INSIGHT

\author{
Helen Parker
}

Pinnacle Ventures. Hamilton. New Zealand

To Cite: Parker H. Establishing patient partners:

The Health Care Home Model. JHD. 2018;3(3):135138. https://doi.org/10.21853/JHD.2018.65

\section{Corresponding Author:}

Helen Parker, Pinnacle Ventures

Level 3, 711 Victoria Street, Hamilton, NZ

helen.parker@ventures.health.nz

\section{Copyright:}

(C) 2018 The Authors. Published by Archetype Health

Pty Ltd.. This is an open access article under the CC BY-NC-ND 4.0 license.

\section{SUMMARY}

The Pinnacle Health Care Home Model redesigns primary care to actively promote engaged consumers and enable patients to have more control over their health care. Changes to achieve this include:

- Active promotion of a patient portal

- Choice of consult mode: 1:1, telephone, email, and shared medical appointments

- Care provided outside of the practice setting

- Practice Patient Participation Groups

- Consumer panel

Successful change relies heavily on clinical leadership and engagement, space for practice teams to plan and review, credible data to track progress, and new roles added to the traditional primary care team.

Key Words

Active consumers; service redesign; portals; redesign

\section{INTRODUCTION}

Development of the Pinnacle Health Care Home $(\mathrm{HCH})$ model for primary care in New Zealand ${ }^{1}$ includes design that enables and promotes patients as active partners in their health care rather than passive recipients. A conceptual framework that underpins the model design has been described as "Patients, families, their representatives, and health professionals working in active partnership at various levels across the healthcare system-direct care, organizational design and governance, and policy making-to improve health and healthcare”. ${ }^{2}$

\section{SUMMARY}

The New Zealand traditional primary care model generally operates a reactive, first come, first served model of care within a universal system of face-to-face, 1:1 consults at a practice, and 15-minute appointments regardless of need and complexity. Within that mix, patients have little space to fully engage as informed consumers of care. Furthermore, a significant minority, but with higher levels of need, are not accessing care at all. Additionally, there is no formal mechanism for patients to influence or co-create service development at the practice or system level.

\section{Heath Care Home model solutions}

The Health Care Home model developed by Pinnacle Inc. in Hamilton, NZ, offers many enhancements to improve access to patient care and to enable patients to be more involved in their health care. These enhancements are outlined below.

1. Offer choice of consult: telephone, email, face-to-face, or group consults with peers.

This option values patient time by offering care, when possible, without the need for a practice visit. Shared medical appointments (SMAs) or peer group consultations for an hour enable patients to have more time for patient education, peer support, and asking questions in an environment maybe less threatening than a practice 1:1 visit. Patient feedback has been very positive in not having to take time out to get to the practice. The SMAs and group 
consultations are a new intervention, but already are achieving improved clinical outcomes with patients reporting better understanding of their conditions and able to self-care more effectively. Clinicians also reported enjoying the new consultation format.

2. Promote the use of the patient portal to enable online access to email, health records, test results, appointment booking, and repeat script ordering.

The use of the portal enables patients to email their general practitioner (GP) and access other services on their own time. GPs anecdotally report patients providing more background information to symptoms or queries than they may on the phone or face-to-face and have not been "deluged" with emails as anticipated.

3. Offer longer appointments for people with more complex needs. Focus groups identified these patients did not feel able to ask questions and understand enough about their condition in 15 minutes.

Practices are offering patients the option of attending a shared medical appointment, which would give them more time with a professional. Practices are also offering longer appointment times for patients who need them.

4. Employing community health workers and taking practice services out of the practice building to where people live, work, and play.

Shifting care to community locations, the workplace, and Māori maraes has enabled clinical teams to work with people who previously have not engaged. The community health workers who are well established in the community networks are able to make connections, build trust, and support clinicians to deliver services in culturally sensitive ways, and in a manner that engages people in their health. Increasingly, our patient stories are revealing better health, improved mental health and wellbeing, and earlier treatment for previously undiagnosed conditions such as diabetes.

5. Establish practice patient participation groups to facilitate discussion in practice service delivery and development.

Practice patient participation groups have enabled patients to have direct communication and debate with GP owners and managers regarding the patient experience at the practice level. Practices have acted on feedback and made changes to practice services, facilities, and communication to patients.

6. Establish a consumer panel to engage, contribute, and provide feedback on strategic service development and health service policy.

This is a new initiative with more than 600 people, representing all demographics, already signed up to be available for targeted focus groups, surveys, service design workshops, and engagement on wider health policy.

7. Create a cloud-based patient information system ${ }^{3}$ that, in addition to creating one shared patient record amongst all providers, enables patients to view their health record, upload data from their wearables or devices, view and update their care or treatment plans, and message members of their care team.

The cloud-based information system provides them with an effective tool to have more control of how they manage their health and health care. 


\section{LESSONS LEARNED}

As the model has evolved through several years of implementation, valuable learning has been gained that has informed model redesign and model development. Important lessons include:

1. The degree to which patients accept and engage with different consult modes and the portal is really dependent on their GP and clinical team having discussions about the benefits to them as a patient/clinician team.

2. Assumptions about what patients want, and how, are often made without any confirming data. Generating data, for example, which shows how many "elderly" patients with complex needs are using online services as well as face-to-face care helps to change traditional custom and practice.

3. Providing care in the right setting for patients does increase engagement and requires time for practice teams to plan and build into the working pattern of the team.

4. Establishing online care requires a redesign of all care pathways to create the necessary clinical capacity to respond. Just adding patient email to an already busy clinical day will make it just busier and unsustainable. Redesigning roles and processes that free up GP time in the day creates this capacity. Adding clinical pharmacists to the team and introducing clinical triage of same-day demand, which reduces the number of face-to-face appointments required, has achieved this but takes time to embed. Targeted change management support and time for practice teams to plan change has been found to be essential.

5. Enhancing the clinical team with well-trained lay members from the local community encourages better access from those who would not normally engage and provides an effective conduit between conversations in the consultation, helping with translation to bring about behavioural change such as diet and exercise.

The outcomes of these, together with other elements of the $\mathrm{HCH}$ model, are very positive as shown by the recent Ernst and Young evaluation. ${ }^{4}$ However, these outcomes are not achieved overnight and it can take a couple of years for both patients and healthcare practitioners to work together more effectively.

\section{DESIGN INSIGHT}

As a general practitioner this Design Insight provides insights on how a large primary care system transitioned from a reactive system without even booked appointments to a Health Care Home model. It is of note that the authors describe this as involving changes in access types, access methods, staffing mix, and technology.

The need for the clinicians to engage in the process of highlighting the benefits of the changes to patients is an effective change management strategy and requires significant understanding of the rationale and support by the clinicians for the changes. One of the lessons learnt is that time and space to redesign processes and systems of care is required, and then ongoing time to plan as a team how to respond to patient needs. A change process or ongoing delivery that does not allow this time and space would struggle.

The authors note that the positive outcomes they refer to took years to achieve. This highlights the importance of realistic timeframes and expectations for all. It is also important to be able to monitor processes, systems, acceptance, and outcomes during implementation, and respond with appropriate adaptions if it becomes apparent an element of the initial plan can be improved.

This type of system would not be possible under some funding models and without autonomy at the level of the service to make significant changes guided by their community needs. 
Dr. Carolyn O'Shea, MBBS, FRACGP, MMed

Deputy Director of Training

Eastern Victoria General Practice Training

Victoria, Australia

General Practitioner, Greensborough Victoria, Australia

\section{REFERENCES}

1. Pinnacle Midlands Health Network. The Health Care Home. [cited 2018 AUG 28]. Available from: http://www.healthcarehome.co.nz

2. CarmanKL, Darless P, Maurer M et al, Patient and Family Engagement: a framework for understanding the elements and developing interventions and policies, Health Affairs (Project Hope). 2013;32:223-31.

3. Indici. [cited 2018 AUG 28]. Available from: https://www.indici.co.nz

4. Health Care Home evaluation-updated analysis, April-September 2017. Auckland: Ernst \& Young, 2018. [cited 2018 AUG 28]. Available from: http://www.healthcarehome.co.nz/wp-content/uploads/2018/05/EY-HCHEvaluation-April-18.pdf

\section{ACKNOWLEDGEMENTS}

None

\section{PEER REVIEW}

Not commissioned. Externally peer reviewed.

\section{CONFLICTS OF INTEREST}

The authors declare that they have no competing interests.

\section{FUNDING}

None

\section{ETHICS COMMITTEE APPROVAL}

None 